Part of Journal of Research of the National Bureau of Standards, Volume 36, April 1946

\title{
A GLOSSMETER FOR SMOOTHNESS COMPARISONS OF MACHINE-FINISHED SURFACES
}

\author{
By Richard S. Hunter
}

\section{ABSTRACT}

As shininess is one indication of surface smoothness, a photoelectric glossmeter was developed for possible use as a production-inspection device for evaluating the roughness of fairly coarse machine-finished surfaces. A near-grazing angle, 75 degrees, was chosen for the measurement of gloss. Therefore, specimens of the type having narrow ridges of metal between adjacent tool cuts are rated low in gloss, or rough. Although the glossmeter essentially measures the fraction of the unshadowed surface that is nearly parallel to mean surface, the instrument resulting from the present development has proved to be a simple and useful device for making rapid comparisons of the roughness of surfaces machined with about the same feeds.

I. Introduction

II. The glossmeter

III. Use of the glossmeter

IV. Comparisons of glossmeter readings with root-mean-square deviations from mean surface.

\section{INTRODUCTION}

Recently the National Bureau of Standards was asked by the War Department to suggest a rapid and simple production-inspection method for comparing the roughness of somewhat similar machinefinished surfaces. The roughness of the surfaces ranged between 100 and 500 microinches root-mean-square deviation from mean surface. The method was needed for identifying the rougher surfaces in this range that could not be rendered smooth by the application of single sprayed films of paint.

As shininess is one indication of surface smoothness, a photoelectric glossmeter was developed and tried for the purpose. A photoelectric reading of the gloss of a surface can be made quickly with this apparatus, which is inexpensive and does not damage the surface. Because the roughness involved is about one-thousand times as large as that which may affect the gloss of surfaces, every test surface has to be coated before gloss measurement with a liquid that will fill its microscopic cracks and pores. Without such a coating, it is impos- 
sible to separate the gloss effects of microscopic irregularities from those of larger irregularities. The choice of a liquid for the purpose is described below.

\section{THE GLOSSMETER}

In planning the glossmeter, a near-grazing angle of specular reflection, 75 degrees, was chosen for two reasons: (1) low-gloss readings (indicative of coarseness) would thereby be given to any specimens having narrow ridges of metal extending appreciably above mean surface, and (2) the specular reflectance of the required coating liquid is much higher at this angle than the specular reflectance of the underlying metal surface covered with liquid; therefore, variation of the gloss of the underlying surface has relatively little effect on glossmeter reading.

In choosing the parts for the apparatus illustrated in figure 1, a barrier-layer photocell was selected because of the simplicity of the electrical equipment required with it. A standard five-cell flashlight lamp, which possesses a small concentrated filament, was chosen for the light source. A $5 / 8$-inch lens having a focal length of $4 \mathrm{~cm}$ was selected because it provides a narrow beam of high flux density.

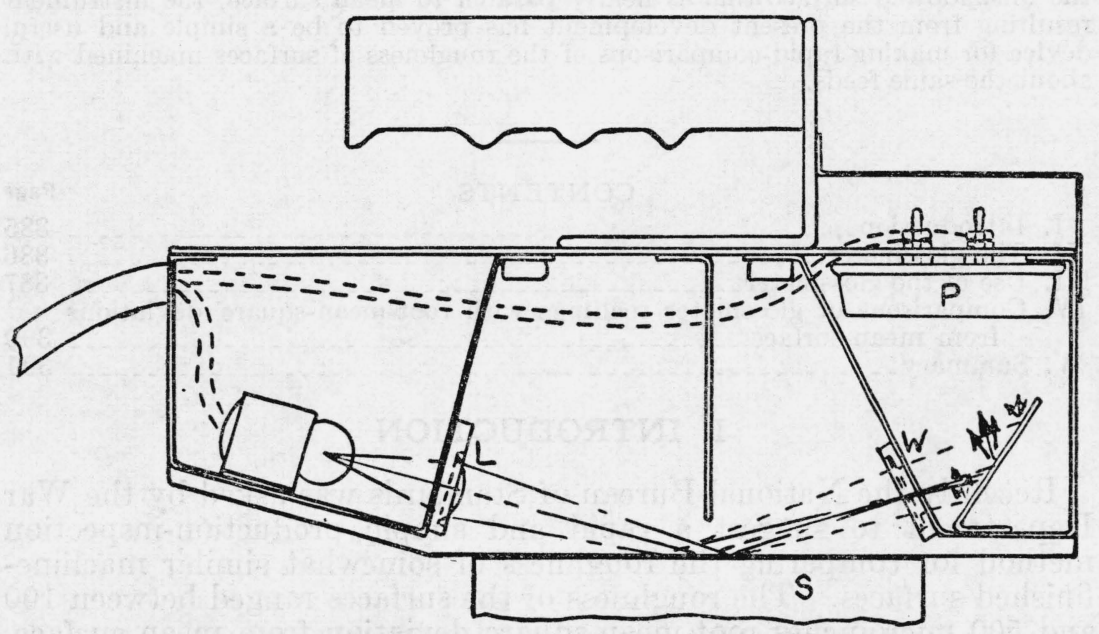

Figure 1.-Vertical cross section of 75-degree glossmeter showing lens $(L)$, specimen in position for measurement $(S)$, window to photocell chamber $(W)$, and barrierlayer photocell $(P)$.

In the glossmeter shown in figure 1, the converging beam from the lens is directed onto a machined surface at right angles to the tool marks. The light specularly reflected by the surface proceeds toward the photocell chamber. The small window at the entrance to this chamber admits only rays of reflected light whose average deviation from the direction of mirror reflection does not exceed 6.5 degrees. The interior of the chamber is coated white to conserve the light that enters.

The body of the instrument is built chiefly of duraluminum. With- 
out handle, it is 25 by 8 by $4 \mathrm{~cm}$ in size. The grip handle makes the unit easy to position on specimens under test.

The control case (fig. 2) that accompanies the glossmeter contains a pointer-type galvanometer for registering photocell current, a 10ohm rheostat for controlling the lamp current, a switch, and a small 110- to 6-volt transformer. As shown in figure 2, room is provided in the case for the glossmeter, connecting cable, and a small bottle of the liquid used to coat test surfaces.

The new glossmeter was designed primarily for measuring surfaces machined by lathe. The beam of light was restricted to small diameter so that test surfaces that are parts of cylinders or cones can be measured. As shown in figure 1 , the plane of the test area must be placed in the plane of the bottom edges of the glossmeter. With cylindrical or conical test specimens of like size and shape, this placement may be conveniently obtained by attaching templates of the proper design to the sides of the glossmeter (see fig. 2).

\section{USE OF THE GLOSSMETER}

Values of 75-degree specular reflectance are proportional to deflections of the galvanometer. Polished black glass is a convenient standard to use with the instrument. The 75-degree specular reflectance of the average piece of black glass is 26 percent. ${ }^{1}$

Liquid-coated steel surfaces of the type being studied were found to reflect from 2 to about 25 percent of the incident beam into the photocell chamber. In using the glossmeter, an operator first places it on a clean, polished black-glass standard and adjusts the rheostat till the galvanometer registers a 26-division deflection. When this adjustment is made, values of specular gloss are read directly as deflections of the galvanometer.

The specular reflectances of 10 steel specimens machined in 8 different shops on lathes using feeds of from 0.017 to 0.025 inch were measured with the glossmeter. The root-mean-square roughness values of the same specimens were measured ${ }^{2}$ with a Profilometer. ${ }^{3}$ The surface of each test specimen was measured with both instruments at four selected areas. The results of these measurements are compared graphically in figures 3 and 4.

It proved difficult to find a coating liquid that would fill microscopic cracks and pores, without filling the larger cavities. The liquid necessarily had to have low surface tension and low viscosity. Kerosine and Stoddard solvent were first tried. A 90-second waiting period was required after Stoddard solvent was brushed generously onto a specimen so that the excess liquid could drain off and leave a film conforming to the major surface irregularities. As shown in figure 3, good separation of the machined surfaces according to their roughness was obtained with Stoddard solvent. Films of kerosine also performed satisfactorily, but a draining period longer than 90 seconds was required.

The draining period required after an application of Stoddard solvent or kerosine is objectionable in a method for production inspec-

${ }^{1}$ D. G. Moore and R. S. Hunter, Use of liquid surafces as standards of specular gloss, J. Am. Ceram. Soc. 24, 167 (1941).

2 By C. E. Haven, of the National Bureau of Standards Gage Section.

'E. J. Abbot, S. Bousky, D. E. Williamson, The Profilometer, Mech. Eng. 60, 205 (1938). 


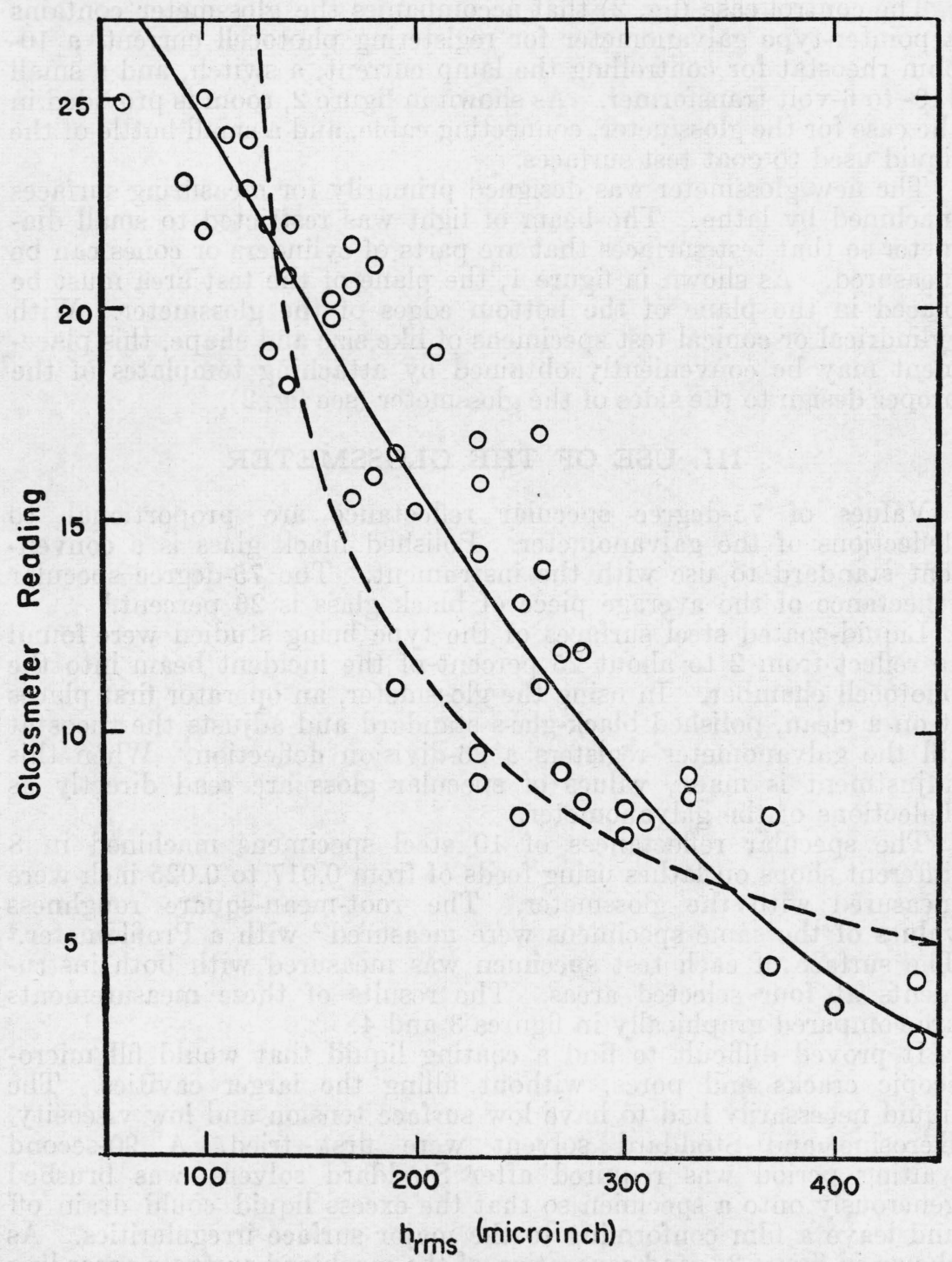

FIGURE 3.-Relation between values of roughness measured with the Profilometer and those of gloss measured with the Stoddard solvent as the coating liquid.

Measurements were made on steel specimens machined in 10 different shops. Dotted line represents relation computed for sine-shaped profile. 


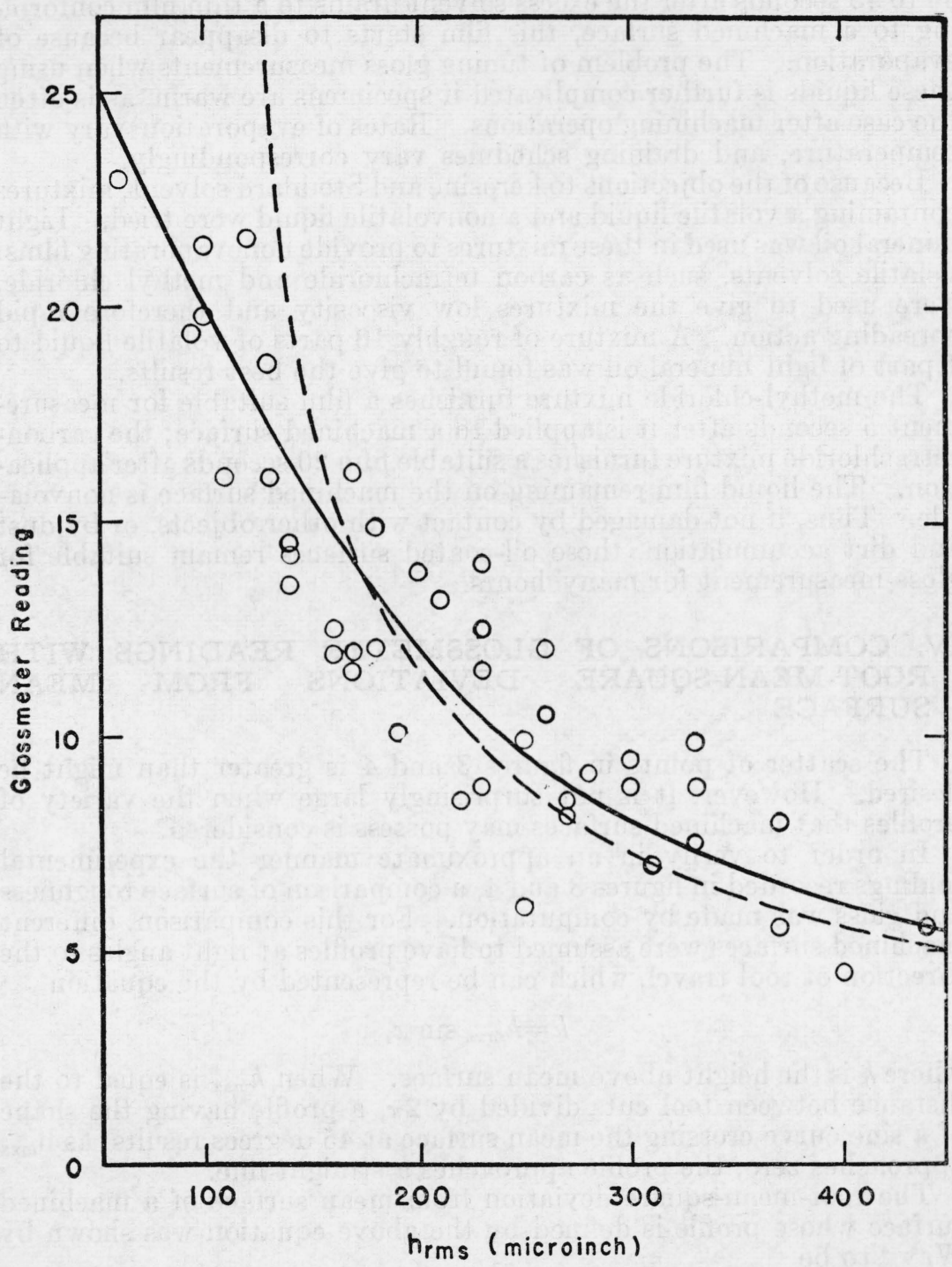

FIGURE 4.-Relation between root-mean-square roughness measured with the Profilometer and gloss measured with mixture of iight mineral oil and carbon tetrachloride as the coating liquids.

Data were obtained from the same test areas as those plotted in figure 3. Dotted line represents relation computed for sine-shaped profile. 
tion. In addition, there is only a brief period after draining in which the gloss values of such coated surfaces remain stable. Thus, about 30 to 45 seconds after the excess solvent drains to a thin film conforming to a machined surface, this film starts to disappear because of evaporation. The problem of timing gloss measurements when using these liquids is further complicated if specimens are warm, as is often the case after machining operations. Rates of evaporation vary with temperature, and draining schedules vary correspondingly.

Because of the objections to kerosine and Stoddard solvent, mixtures containing a volatile liquid and a nonvola tile liquid were tried. Light mineral oil was used in these mixtures to provide nonevaporating films; volatile solvents, such as carbon tetrachloride and methyl chloride, were used to give the mixtures low viscosity and therefore rapid spreading action. A mixture of roughly 10 parts of volatile liquid to 1 part of light mineral oil was found to give the best results.

The methyl-chloride mixture furnishes a film suitable for measurement 5 seconds after it is applied to a machined surface; the carbontetrachloride mixture furnishes a suitable film 20 seconds after application. The liquid film remaining on the machined surface is nonvolatile. Thus, if not damaged by contact with other objects, or by dust and dirt accumulation, these oil-coated surfaces remain suitable for gloss measurement for many hours.

\section{COMPARISONS OF GLOSSMETER READINGS WITH ROOT-MEAN-SQUARE DEVIATIONS FROM MEAN SURFACE}

The scatter of points in figures 3 and 4 is greater than might be desired. However, it is not surprisingly large when the variety of profiles that machined surfaces may possess is considered.

In order to verify in an approximate manner the experimental findings recorded in figures 3 and 4, a comparison of surface roughness and gloss was made by computation. For this comparison, different machined surfaces were assumed to have profiles at right angles to the direction of tool travel, which can be represented by the equation

$$
h=h_{\max } \sin x,
$$

where $h$ is the height above mean surface. When $h_{\max }$ is equal to the distance between tool cuts divided by $2 \pi$, a profile having the shape of a sine curve crossing the mean surface at 45 degrees results; as $\mathrm{h}_{\mathrm{mxa}}$ approaches zero, the profile approaches a straight line.

The root-mean-square deviation from mean surface of a machined surface whose profile is defined by the above equation was shown by Way ${ }^{4}$ to be

$$
h_{\mathrm{rms}}=0.708 h_{\max } \text {. }
$$

The value of specular gloss obtained from a machined surface with the present glossmeter is determined by the fraction of the surface reflecting light in to the photocell chamber. The glossmeter was built to accept all light reflected within 6.5 degrees of the direction of

\footnotetext{
- S. Way, Description and observation of metal surfaces, Proc. special summer conference on friction and surface finish, p. 44, Massachusetts Institute of Technology, 1940. (Published by Murray Printing O0., Cambridge, Mass.).
} 
reflection from a smooth surface. Because a reflected beam rotates twice as fast as the surface which reflects it, the only areas of a test surface that can thus direct light into the photocell chamber are those less than 3.25 degrees from parallel to the mean surface.

The fraction of the incident beam that is reflected into the photocell chamber by a machined surface measured across the direction of tool travel can be shown from the above considerations to be

$$
R=\left(1-\frac{2}{\pi} \cos ^{-1} 0.0064 \lambda / h_{\mathrm{rms}}\right) R_{\mathrm{max}},
$$

where $\lambda$ is the feed, or distance between tool cuts, and $R_{\max }$ is the reflectance that the coated metal surface would have if flat. The tool feeds for the steel specimens used in this study were about 0.020 inch. The glossmeter readings for the smooth steel specimens were found always to be 25 or 26 . For these reasons, $\lambda$ was made 0.020 inch and $R_{\max }$ was made 26 in computing the expected relation between gloss and $h_{\mathrm{rms}}$. This relation is shown graphically by the dotted-line curve in figures 3 and 4 . When the probable differences between actual profiles and assumed profiles are considered, the agreement between computed and observed data is considered reasonable.

\section{SUMMARY}

A glossmeter has been developed for use in comparing the roughness of machined surfaces. Although this instrument actually evaluates that fraction of surface area nearly parallel to mean surface not shaded by peaks, the instrument gives rapid and reasonably reliable comparisons of the roughness of different surfaces prepared with about the same tool feeds. Because glossmeter readings depend on the profile shapes rather than profile dimensions, the glossmeter is not a suitable instrument for roughness comparisons where tool feeds differ appreciably from specimen to specimen.

WASHington, January 7, 1946. 




Figure 2.-Glossmeter on specimen, and control box. 